The potential of ge-doped optical fibre TL dosimetry for $3 \mathrm{D}$ verification of high energy IMRT photon beams

Noramaliza M. Noor, M. Hussein, D.A. Bradley, A. Nisbet

PII: $\quad$ S0168-9002(10)00041-0

DOI: $\quad$ doi:10.1016/j.nima.2010.01.013

Reference: $\quad$ NIMA 50998

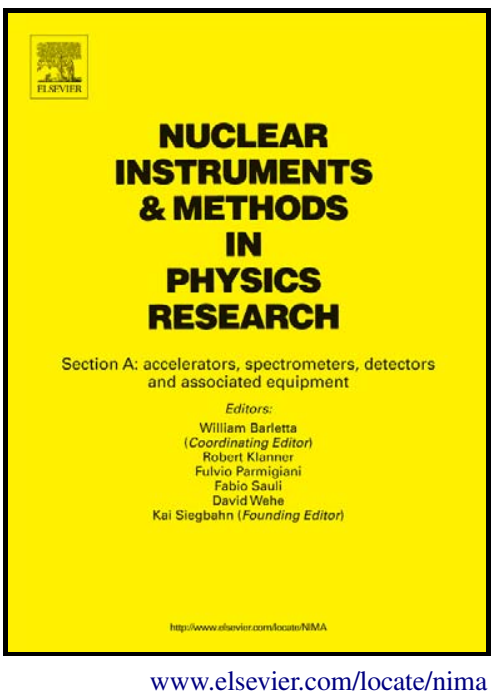

To appear in: Nuclear Instruments and Methods in Physics Research A

Received date: 20 August 2009

Revised date: 6 January 2010

Accepted date: 8 January 2010

Cite this article as: Noramaliza M. Noor, M. Hussein, D.A. Bradley and A. Nisbet, The potential of ge-doped optical fibre TL dosimetry for 3D verification of high energy IMRT photon beams, Nuclear Instruments and Methods in Physics Research A, doi:10.1016/j.nima.2010.01.013

This is a PDF file of an unedited manuscript that has been accepted for publication. As a service to our customers we are providing this early version of the manuscript. The manuscript will undergo copyediting, typesetting, and review of the resulting galley proof before it is published in its final citable form. Please note that during the production process errors may be discovered which could affect the content, and all legal disclaimers that apply to the journal pertain. 


\title{
The Potential of Ge-Doped Optical Fibre TL Dosimetry for 3D Verification of High Energy IMRT Photon Beams
}

\author{
Noramaliza M.Noor ${ }^{1 *}$, M Hussein ${ }^{2}$, D.A. Bradley ${ }^{1}$ and A. Nisbet ${ }^{1,2}$ \\ ${ }^{1}$ Centre for Nuclear and Radiation Physics, Department of Physics, University of Surrey, \\ Guildford, GU2 7XH, UK \\ ${ }^{2}$ Department of Medical Physics, St Luke's Cancer Centre, The Royal Surrey County Hospital \\ NHS Trust, Edgerton Road, Guildford, GU2 7XX, UK \\ *Author for correspondence: n.mohdnoor@surrey.ac.uk
}

\begin{abstract}
Intensity Modulated Radiation Therapy (IMRT) is a technique widely used in the treatment of patients with prostate cancer, the most commonly occurring male cancer in the United States and Western Europe. The technique has many attractive features, promising improved radiotherapy over that provided by conventional techniques, including enabling the tumour to be treated with a uniform high dose, capability for shaping the radiation beams to match the shape of the tumour and potentially improving patient outcome. However, there are a number of concerns involving high photon energy IMRT ( $>10 \mathrm{MV})$, including greater radiation leakage and the possibility of photo-neutron production. The aim of the present study is to investigate the potential of Ge-doped optical fibre thermoluminescent (TL) dosimetry in determining typical out-of-field doses for high energy IMRT. Commercial Ge-doped optical fibres have been employed as the TL dosimeters, offering features such as high sensitivity, cost-effectiveness and small size. Extensive measurements have been made, examining reproducibility, sensitivity, energy response and linearity with dose. Screening for sensitivity to dose of the individual fibres and subsequent selection has led to an overall coefficient of variation $(\mathrm{CV})$ of better than $4 \%$. Use has been made of an anthropomorphic phantom (RANDO) for photon irradiation measurements delivered over the range of nominal energies 6 - 15 MV as typically used in IMRT treatment of prostate cancer. Comparison has been made with TLD-100 measurements, the latter being corrected at $15 \mathrm{MV}$ for their response to thermal neutrons. The study has demonstrated the Ge-doped optical fibre thermoluminescent (TL) dosimeters to offer good potential for use in IMRT radiotherapy when using $6 \mathrm{MV}$ photons, also indicating a need to correct their response to neutrons when conducting $15 \mathrm{MV}$ irradiations.
\end{abstract}

\section{Keywords: IMRT, Ge-doped optical fibres, RANDO phantom, TLD-100 and TLD-700 INTRODUCTION}

Advanced delivery techniques in radiotherapy such as IMRT have developed rapidly. However, despite the increased dosimetric benefits of IMRT there are still concerns with regard to the use of IMRT due to the increased number of monitor units required and the potential for secondary cancer induction [1]. This is particularly true for high photon energy $(>10 \mathrm{MV})$ IMRT where photo-neutron production becomes a reality [2]. The issue becomes of increased concern for out-of-field irradiations, where for photon energies $>10 \mathrm{MV}$, the relative contribution of neutron dose to the overall dose increases. Thus for instance, measurements of the photon dose component far from the treatment field, determined by TLD-100, have been observed to be highly inaccurate at $18 \mathrm{MV}$ [3].

In radiotherapy treatment, it is crucial to verify the dose distribution before any treatment, due to the possible biological complications of the dose delivered. Moreover, it would also be 
impractical to measure dose distribution directly in patients treated with radiation. Therefore there is a need for dosimetry systems which offer features that may accurately verify the delivered dose to the patient. These include high spatial resolution, accurate and precise dose measurement and tissue equivalence. This study investigates the potential of Ge-doped optical fibre. For TL measurements it has been previously established that the use of Ge-doped optical fibre offers superior performance to other types of commercially produced optical fibre, providing in particular a wide range of dose sensitivity. Other factors that favour use of optical fibres for radiation dosimetry include their small physical size, the absence of cables and associated high voltages and the capability to provide excellent TLD characteristics [4]. The Ge-doped fibre TL system is compared with the more commonly used lithium fluoride material, TLD-100. TLD-100 has a ${ }^{6} \mathrm{Li}$ content of $7.5 \%$ and a ${ }^{7} \mathrm{Li}$ content of $92.5 \%$ whereas the more expensive TLD-700 has respective contents of $0.01 \%$ and $99.99 \%$. As ${ }^{6} \mathrm{Li}$ has a high thermal neutron cross section its use in higher energy beams is limited but TLD-100 could be used if suitable corrections were to be made for neutron dose [5].

\section{MATERIALS AND METHODS}

\subsection{Ge-doped Optical fibres}

Use has been made of single mode commercial Ge-doped silica glass optical fibres (INOCORP, Canada) with a core diameter of $9 \mu \mathrm{m}$ and a cladding diameter of $116 \pm 0.1 \mu \mathrm{m}$. There are four essential steps in preparing the optical fibres prior to irradiation. This includes removal of the outer polymer of the fibre, cleansing of the residual fibre with methyl alcohol, cutting the fibre into convenient lengths to form individual dosimeters $(6 \mathrm{~mm}$ long strands in the present case) and annealing. A detailed description of the procedure is given by Hashim et al. [6].

\subsubsection{Screening process}

Prior to use in clinical applications each batch should be screened to ensure the selection of dosimeters having approximately equal mass, length and sensitivity, discarding strands which do not conform to the prescribed dimensions or that have sensitivity outside a defined range of the mean [7]. For this purpose, each batch of optical fibres was irradiated to a photon component of dose of 3 Gy using a $15 \mathrm{MV}$ photon beam delivering dose at the rate of 400 $\mathrm{cGy} / \mathrm{min}$; a focus to surface distance (FSD) of $100 \mathrm{~cm}$ and $10 \times 10 \mathrm{~cm}^{2}$ field size were also used in delivering these irradiations. After irradiation the optical fibres were kept in a light-tight box for 12 hours to allow uniform control of thermal fading prior to read out of the TL yield using a Solaro TL reader (Vinten TLD, Reading UK). Finally, a distribution of TL yield (corrected counts per second per unit mass of fibre) against the complete set of irradiated optical fibres was produced and analysed. A limit of $4 \%$ in the coefficient of variation (CV) was applied in carrying out the selection process. The CV was observed to be $33.8 \%$ prior to the selection, decreasing to $3.96 \%$ after selection (Figures 1 and 2). A total of 500 fibres were eventually produced as a result of such screening to allow for the investigations reported below.

Figure 1. Prior to sensitivity selection the coefficient of variation (CV) of fibres was found to be $33.8 \%$. A total of 407 fibres were irradiated in the run that is represented by the present set of data.

Figure 2. Subsequent to sensitivity selection the coefficient of variation (CV) of fibres was found to be $3.96 \%$. Following selection, a total of 135 fibres were left from the original number of 407 . 


\section{$2.2 T L D-100$}

To allow comparison of the Ge-doped optical fibres against a better established TL system, use has been made of TLD-100 chips and disks. The chips were of dimension $3.2 \times 3.2 \times 0.89$ $\mathrm{mm}$ while the disks were of $4.5 \mathrm{~mm}$ diameter $\times 0.89 \mathrm{~mm}$ thick, all calibrated against a Farmer-type ionisation chamber. The TLDs were read-out using a QADOS Harshaw 5500 reader with pre-heat at $145^{\circ} \mathrm{C}$ for 10 seconds and a temperature ramp of $17{ }^{\circ} \mathrm{C}$ per second up to a maximum temperature of $300{ }^{0} \mathrm{C}$ for 10 seconds. The response of these to neutrons resulting from $15 \mathrm{MV}$ x-ray irradiation was corrected using the results of Kry et al [5], the latter comparing TLD-100 with TLD-700.

\subsection{Ge-doped dose calibration}

Dose calibration was performed by irradiating the selected Ge-doped optical fibres with $6 \mathrm{MV}$ and $15 \mathrm{MV}$ energy photon beams (using a Varian Linear accelerator and $100 \mathrm{~cm} \mathrm{FSD,} 10 \times 10$ $\mathrm{cm}^{2}$ field size) at $400 \mathrm{cGy} / \mathrm{min}$ dose-rate. The fibres were irradiated with doses ranging from 0.2 cGy up to 100 cGy. A linear relationship between TL-yields and dose (cGy) for both 6 $\mathrm{MV}$ and $15 \mathrm{MV}$ energies was observed (Fig 3).

Figure 3. Calibration curve for Ge-doped optical fibres for $6 \mathrm{MV}$ (left hand graph) and $15 \mathrm{MV}$ (right hand graph) photon beam irradiations.

\subsection{Alderson RANDO anthropomorphic phantom and measurements}

For this project, use was made of a RANDO phantom (The Phantom Laboratory, Salem, NY) version ARTF 1025, with height $1.55 \mathrm{~m}$, weight $50 \mathrm{~kg}$ and transverse sections (of which there are 31 in all) $2.5 \mathrm{~cm}$ thick, each section containing a matrix of $5 \mathrm{~mm}$ diameter holes, depth 2.5 $\mathrm{cm}$, spaced at $1.5 \mathrm{~cm} \times 1.5 \mathrm{~cm}$ to accommodate tissue-equivalent and lung tissue-equivalent plugs. The plugs can be replaced by TLD-100 and Ge-doped optical fibre holders (Figure 4a). Measurements were made as a function of distance along the z-axis of the phantom, away from the Planning Target Volume (PTV), the latter being understood to be located about the central axis of the beam. For a detailed definition of PTV see ICRU Report 62 [8].

\subsection{Treatment planning and irradiation}

Individual IMRT verification plans were produced for the RANDO phantom, based on data from two patients with pathologically diagnosed prostate cancers of different prostate volume. IMRT five-field verification plans were produced for each set of patient data and each plan was used for both the $6 \mathrm{MV}$ and $15 \mathrm{MV}$ photon irradiations. Figure $4 \mathrm{~b}$ shows an example of one such plan. A total of eight phantom measurements were performed, four using the Gedoped fibres, to accommodate the two cases for the two energies, and an equivalent four for the LiF dosimeters.

\subsubsection{Typical Out-of-field doses}

Typical in-field and out-of-field dose measurements (the latter meaning dose measurements made outside of the PTV) were carried out using approximately 130 TLD-100 and 500 Gedoped optical fibres distributed along the length of the RANDO phantom at two specific $(x, y)$ co-ordinates, the location of the x-position changing with the lateral extent of the RANDO phantom. All irradiations were performed using the same Varian Linear Accelerator as 
discussed above, doses being delivered at $6 \mathrm{MV}$ and $15 \mathrm{MV}$ only for the IMRT verification plans. As inferred above, the phantom was positioned on the patient couch of the Varian Linear accelerator (Figure 4c) and irradiations were made using an FSD of $89 \mathrm{~cm}$.

(a)

(b)

(c)

Figure 4. (a) Ten optical fibres were contained in each black plastic rod holder, subsequently inserted into specific holes to replace the tissue-equivalent plugs (b) The IMRT prostate verification plan for the RANDO phantom (consisting of 5 radiation fields) (c) Set-up of the phantom on the Varian Linear Accelerator.

\section{RESULTS AND DISCUSSION}

For $6 \mathrm{MV}$ irradiations, Figures 5 and 6 show the average absorbed doses measured by the optical fibres to be in general accord with TLD-100 values, the error bars representing the standard error of the mean reading of the several dosimeters in that position. For both the in-field and out-of-field evaluations no difference is observed between the Ge-doped fibre and TLD-100 measurements; statistical analysis showing differences between the average dose measured using TLD-100 and optical fibres to be insignificant $[\mathrm{p}=0.532$ and 0.206 for the individual IMRT verification plans 1 and 2 respectively].

For $15 \mathrm{MV}$ irradiations, as expected, a large significant difference is to be noted between TLD-100 (both corrected and uncorrected for neutron dose; see below) and optical fibre measurements for both sets of plans for out-of-field measurements, a situation that is not observed for in-field measurements (Figure 7 and 8). The p-value for the out-of-field measurements was found to be significant at $<0.05$. Here it is to be noted that TLD-100 contains $7.5 \%{ }^{6} \mathrm{Li}$, the thermal neutron absorption cross-section of which is large, $\sim$ a few thousand barn [9,10], influencing the average dose measurements to a considerable extent. The estimates of discrepancies as observed by Kry et al. [5] have been used to offset the present TLD-100 results for the effects of neutron dose, as shown in the two figures. Conversely, the thermal neutron absorption cross-section of the major constituents of the optical fibre, germanium and silica, are both relatively small at $\sim 10$ barn and $\sim$ 1 barn respectively [10], as also discussed in Ref. [6]. The Ge-doped fibre results have not been corrected for neutron dose.

Figure 5. Average doses obtained for 6 MV irradiations, measured using TLD-100 and Gedoped optical fibres for Plan 1 for a PTV of $104.1 \mathrm{~cm}^{3}$. The TLD-100 and Ge-doped optical fibres response at high doses (from $2300 \mathrm{mGy}$ to $40 \mathrm{mGy}$ ) measured up to $10 \mathrm{~cm}$ from the PTV are shown within the inset figure in figure 5. The inset figure has the same units as the main figure .

Figure 6. Average doses obtained for $6 \mathrm{MV}$ irradiations, measured using TLD-100 and Gedoped optical fibres for Plan 2 for a PTV of $79.7 \mathrm{~cm}^{3}$. The TLD-100 and Ge-doped optical 
fibre response at high doses from (1990 mGy to $30 \mathrm{mGy}$ ) measured up to $10 \mathrm{~cm}$ from the PTV are shown as inset in the figure 6 . The inset figure has the same units as the main figure .

Figure 7. Average doses obtained for $15 \mathrm{MV}$ irradiations, the graph showing TLD-100 (uncorrected) and TLD-100 (corrected for thermal neutron dose), compared to Ge-doped optical fibre for Plan 1. The TLD-100 and Ge-doped optical fibre response at high doses from (2000 mGy to $20 \mathrm{mGy}$ ) are shown as inset in the figure 7 measured up to $10 \mathrm{~cm}$ from the PTV. The inset figure has the same units as the main figure. The in-field doses being apparent that here the contribution from the neutron dose component to the overall dose is relatively unimportant.

Figure 8. Average doses obtained for $15 \mathrm{MV}$ irradiations, the graph showing TLD-100 (uncorrected) and TLD-100 (corrected for thermal neutron dose), compared to Ge-doped optical fibre for Plan 2. The TLD-100 and Ge-doped optical fibre response at high doses from (1211 mGy to $30 \mathrm{mGy}$ ) are shown as inset in the figure 8 measured up to $10 \mathrm{~cm}$ from the PTV. The inset figure has the same units as the main figure. Again, the in-field doses being apparent that here the contribution from the neutron dose component to the overall dose is relatively unimportant.

\section{CONCLUSION}

Ge-doped optical fibres show considerable promise as high spatial resolution dosimeters, being capable of determining typical in and out-of-field doses for $6 \mathrm{MV}$ energy photon beams. The indose measurements at $6 \mathrm{MV}$ and $15 \mathrm{MV}$ both show there to be an inappreciable contribution to the overall dose from the thermal neutron component, as expected. Conversely, for out-of-field measurements a highly significant contribution of thermal neutrons to the overall dose is observed. As a result of the finite but relatively small Ge neutron absorption cross-section for photon energies above the photo-neutron production threshold ( $\sim 10 \mathrm{MV})$, it is clear that the optical fibres do overrespond as a result this photo-neutron component. Further investigations concerning the magnitude of this effect are in progress.

\section{ACKNOWLEDGMENTS}

Use of irradiation facilities at the Royal Surrey County Hospital is acknowledged. Also acknowledged are the University of Science of Malaysia and the Ministry of Higher Education, Malaysia for sponsoring the first author under the Academic Staff Training Scheme (ASTS).

\section{REFERENCES}

[1] Welsh, J.S., Mackie, T.R., Limmer, J.P., 2007. High-energy Photons in IMRT:Uncertainties and Risks for Questionable Gain. Technology in Cancer Research and Treatment. 6,147-149.

[2] Followill, D.S., Nusslin, D.F., Orton, C.G., 2007. IMRT should not administered at photon energies greater than $10 \mathrm{MV}$. Med.Phys. 34, 1877-1879.

[3] Sneed, P.K, Albright, N.W., Wara W.M., Prados, M.D., Wilson, C.B., 1995. Fetal dose estimates for radiotherapy of brain tumors during pregnancy. Int J Radiat Oncol Biol Phys. 32, 823-830.

[4] Hashim, S., Al-Ahbabi, S., Bradley D.A., Webb. M, Jeynes, C., Ramli, A.T., Wagiran, H., 2009. The thermoluminescence response of doped $\mathrm{SiO} 2$ optical fibres subjected to photon and electron irradiations. Applied Radiation and Isotopes. 67, 423-427. 
[5] Kry F.S., Salehpour, M., Titt, U., White, R.A.,Stovall, M., Followill, D., 2007. The use $\mathrm{LiF}(\mathrm{TLD}-100)$ as an out-field dosimeter. Journal of Applied Clinical Medical Physics. 8, 167174.

[6] Hashim, S., 2009. The Thermoluminescence Response of Doped Silicon Dioxide Optical Fibres to Ionizing Radiation, Universiti Teknologi Malaysia. PhD Thesis (unpublished).

[7] Furetta, C., 2003. Handbook of Thermoluminescence, World Scientific Publishing Co.Pte.Ltd.

[8] International Commission on Radiation Units (ICRU), 1999, Prescribing, Recording and Reporting of Photon Beam Therapy (ICRU Report 62, Supplement to ICRU Report 50), (Bethesda, USA).

[9] Ermolaev, O.P., 1997. Low-temperature photoluminescence in germanium irradiated by fast neutrons. Journal of Applied Spectroscopy. 64, 493-496.

[10] Nuclear Data Evaluation Lab., 2008. Korean Atomic Energy Research Institute. http://atom.kaeri.re.kr/cgi-bin/endfplot.pl. Accessed on $14^{\text {th }}$ August 2009. 


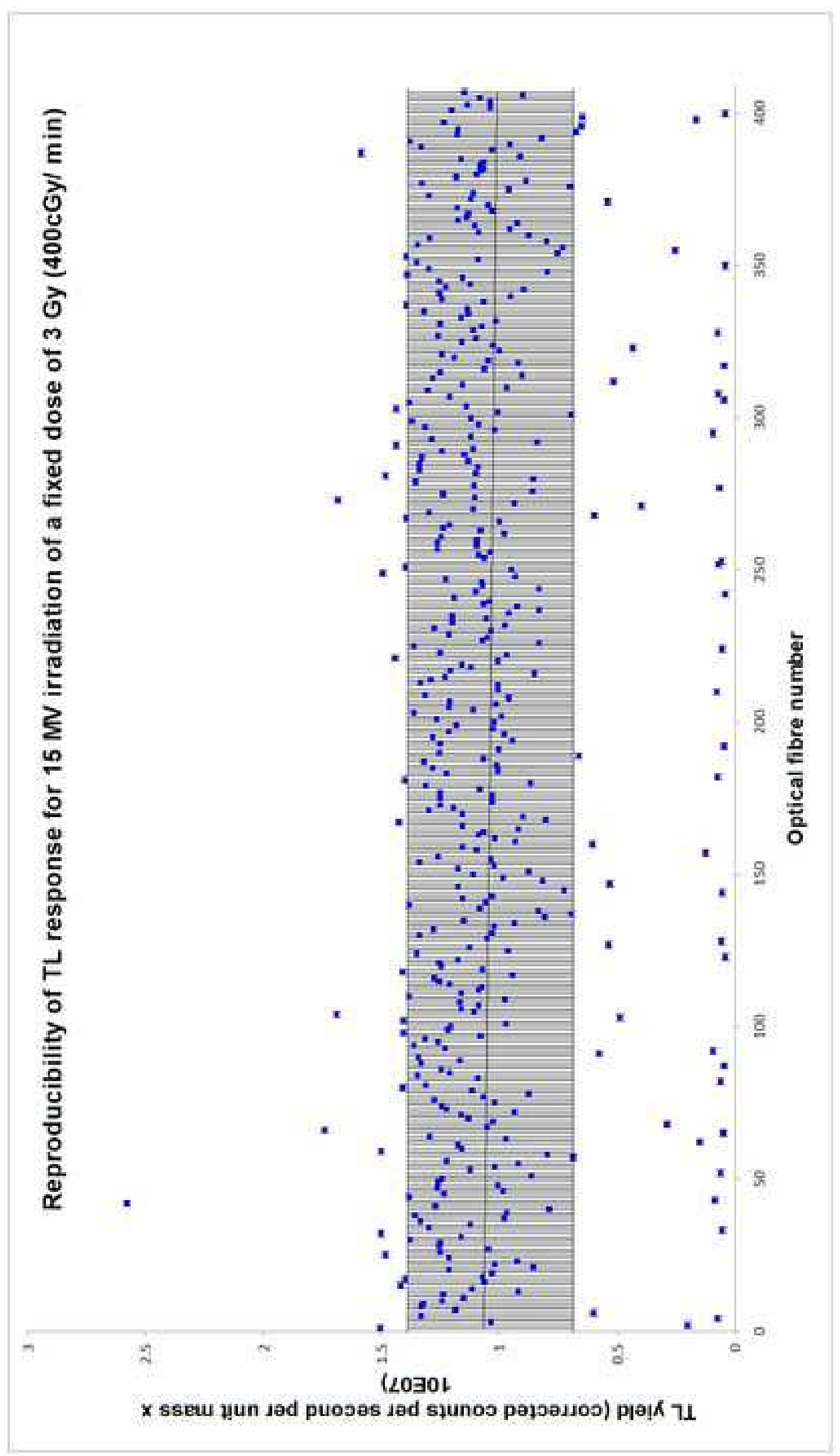




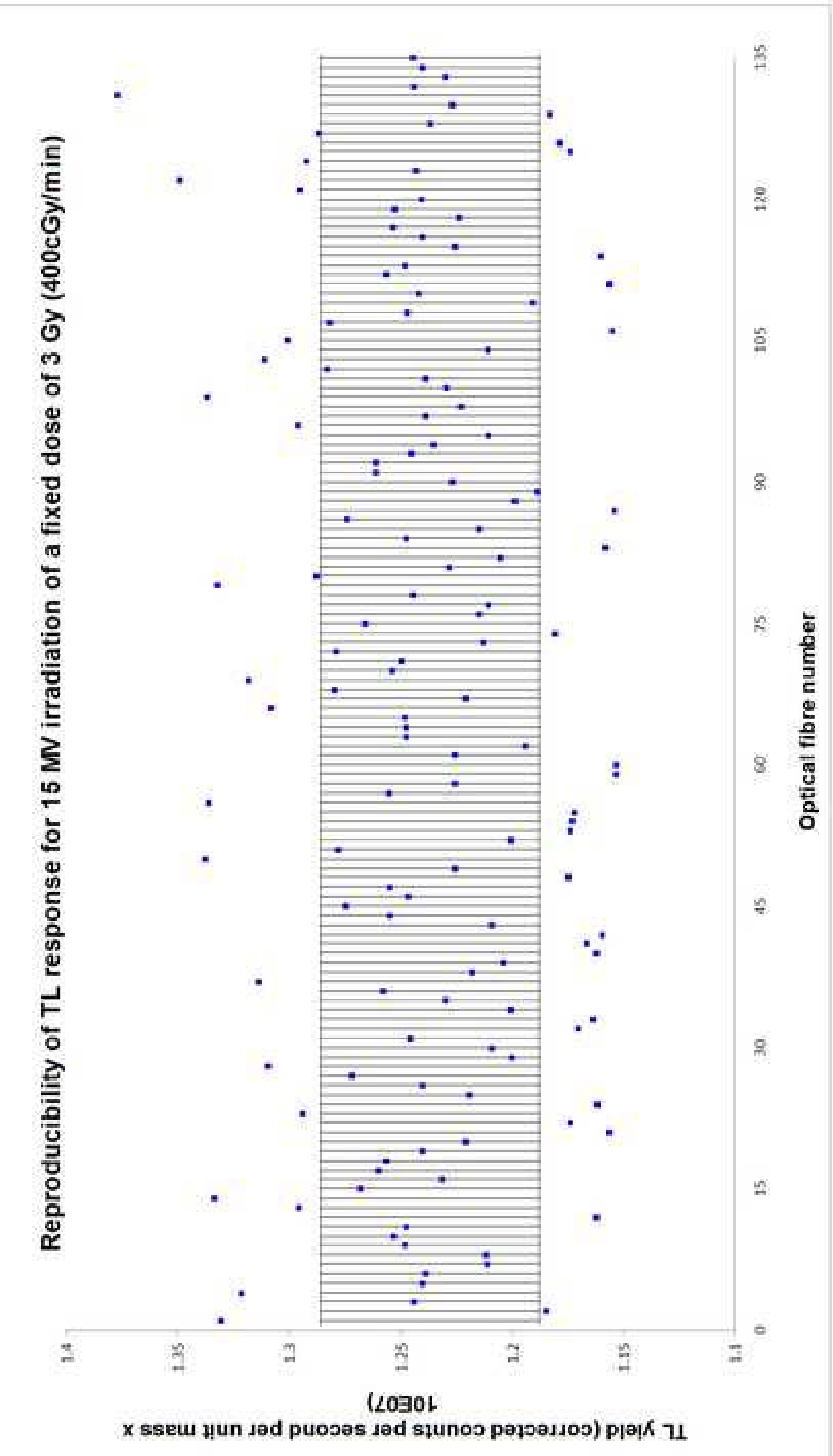




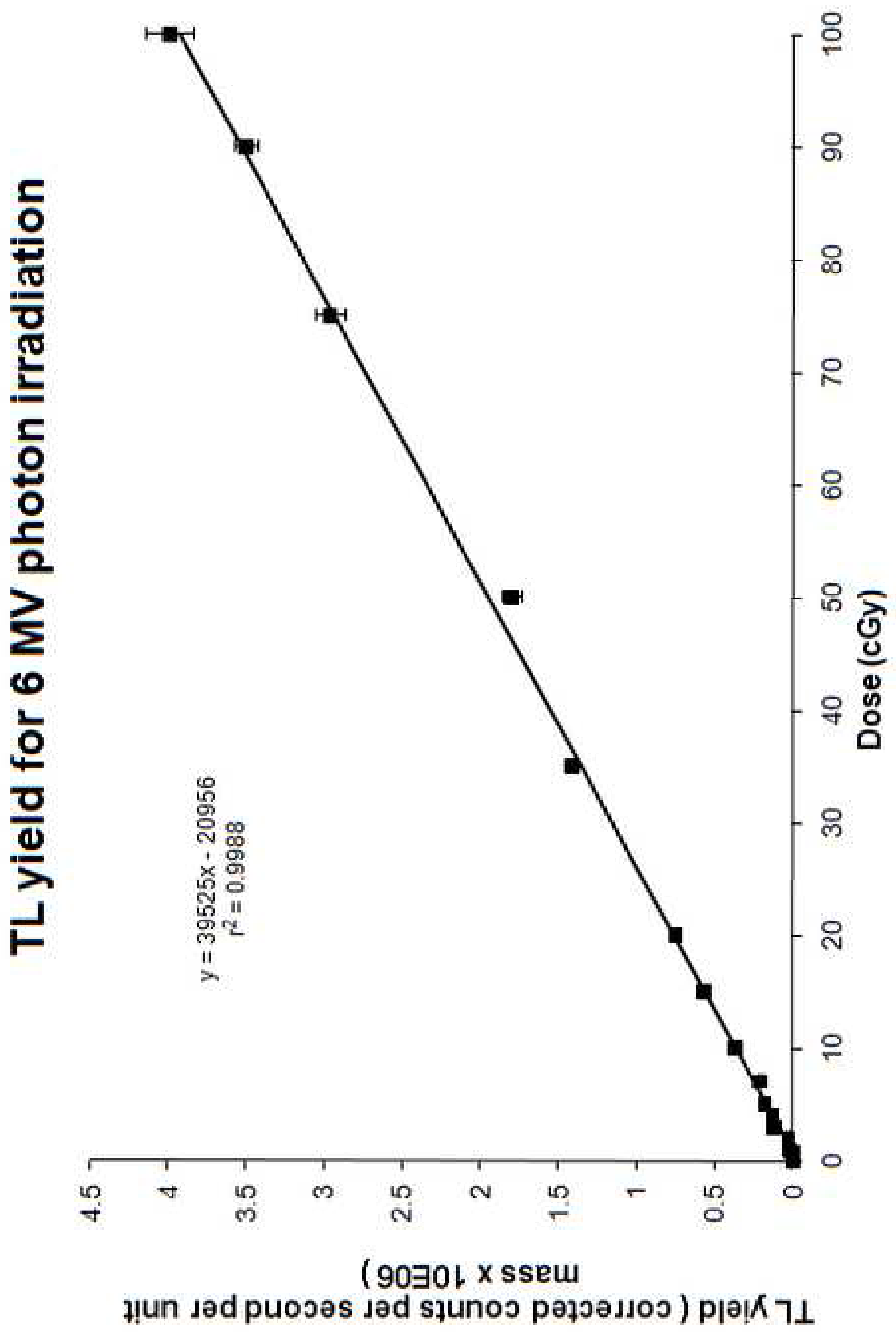




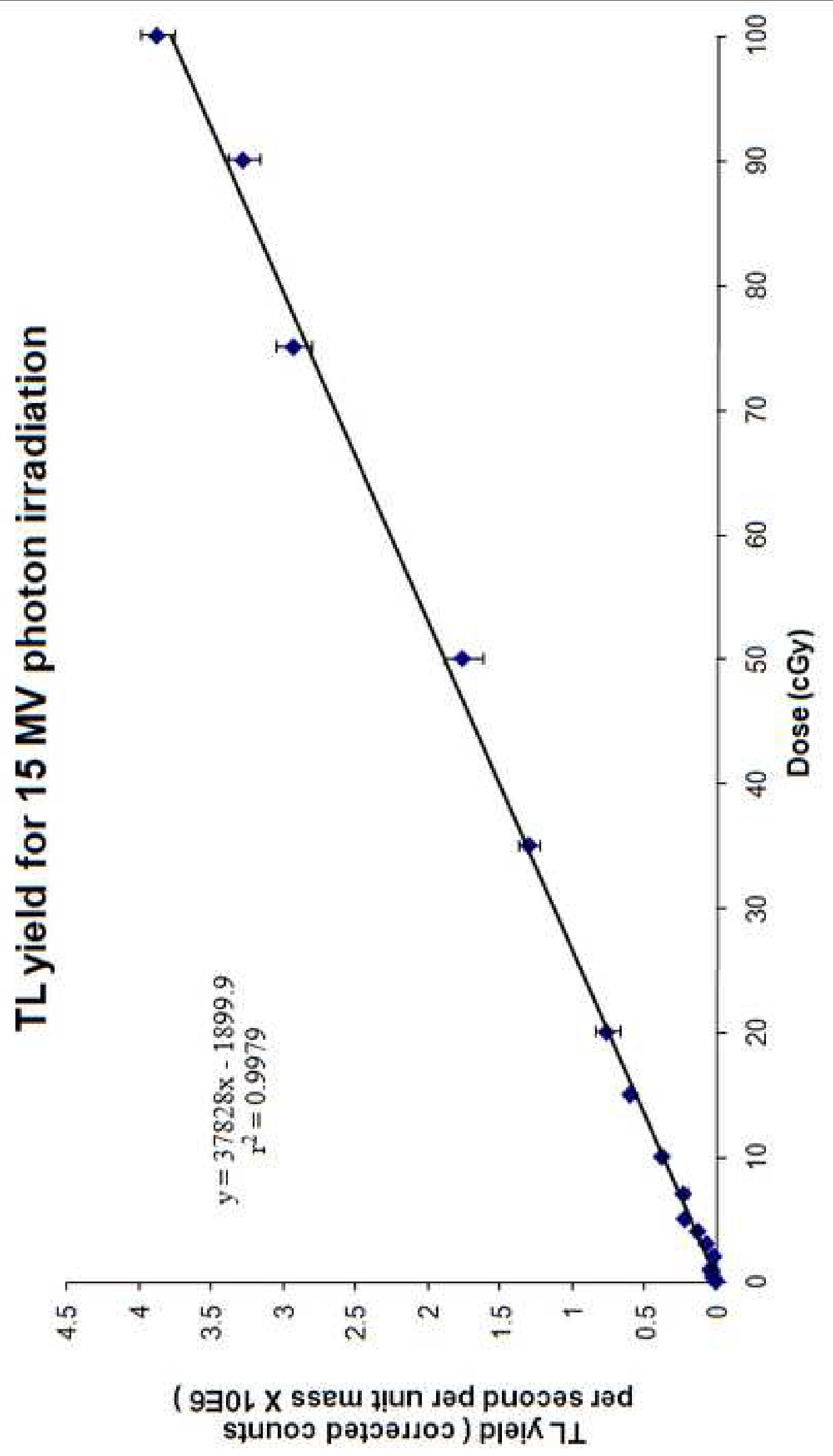




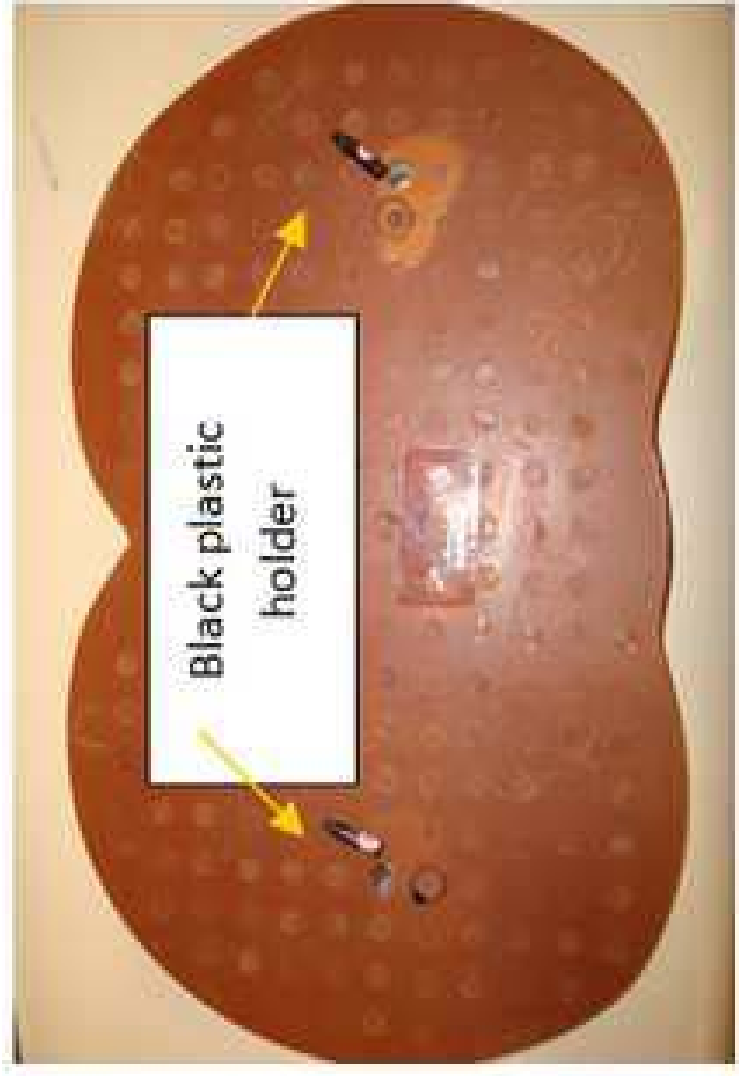

은 


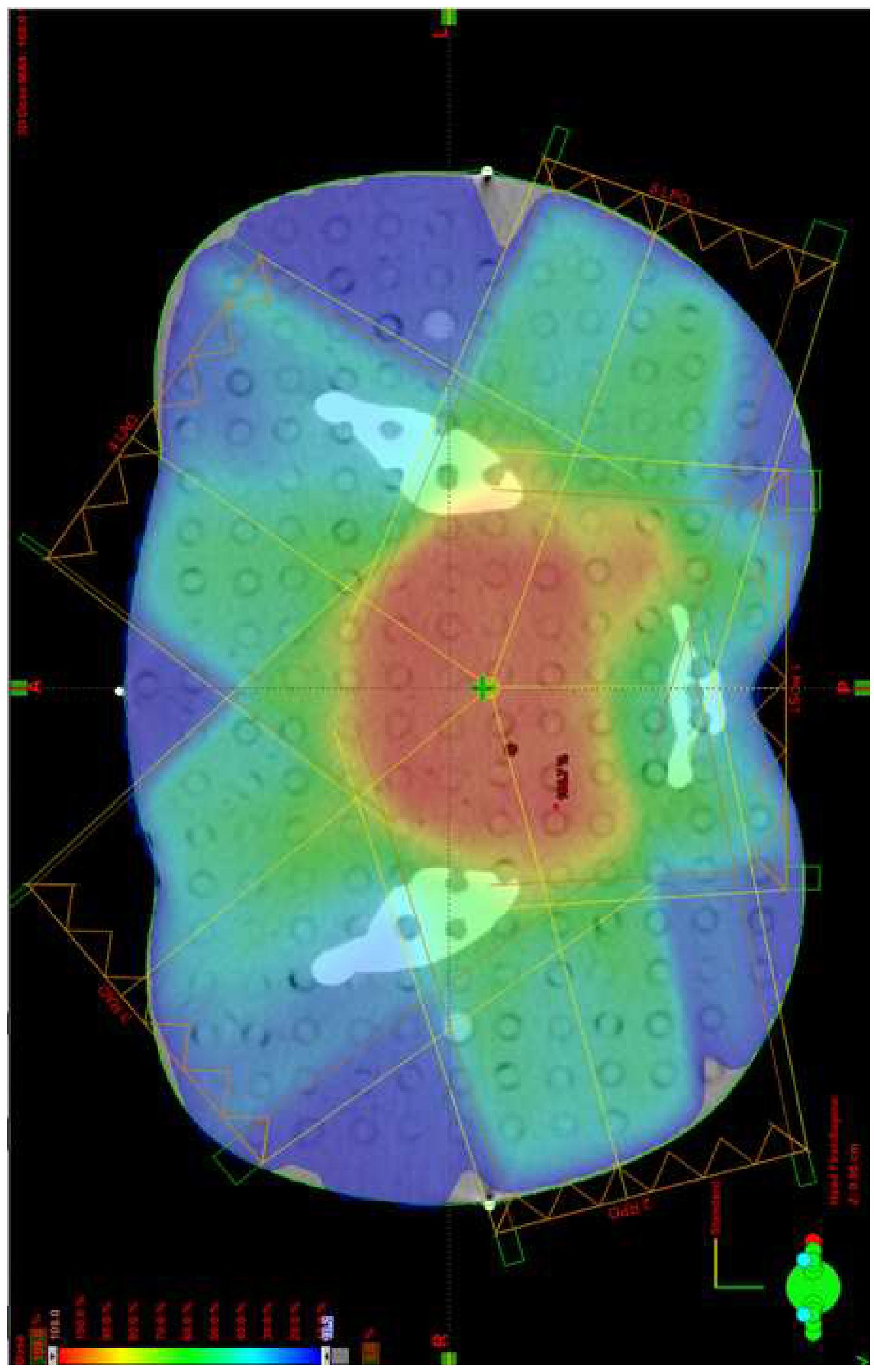


ACCEPTED MANUSCRIPT

D

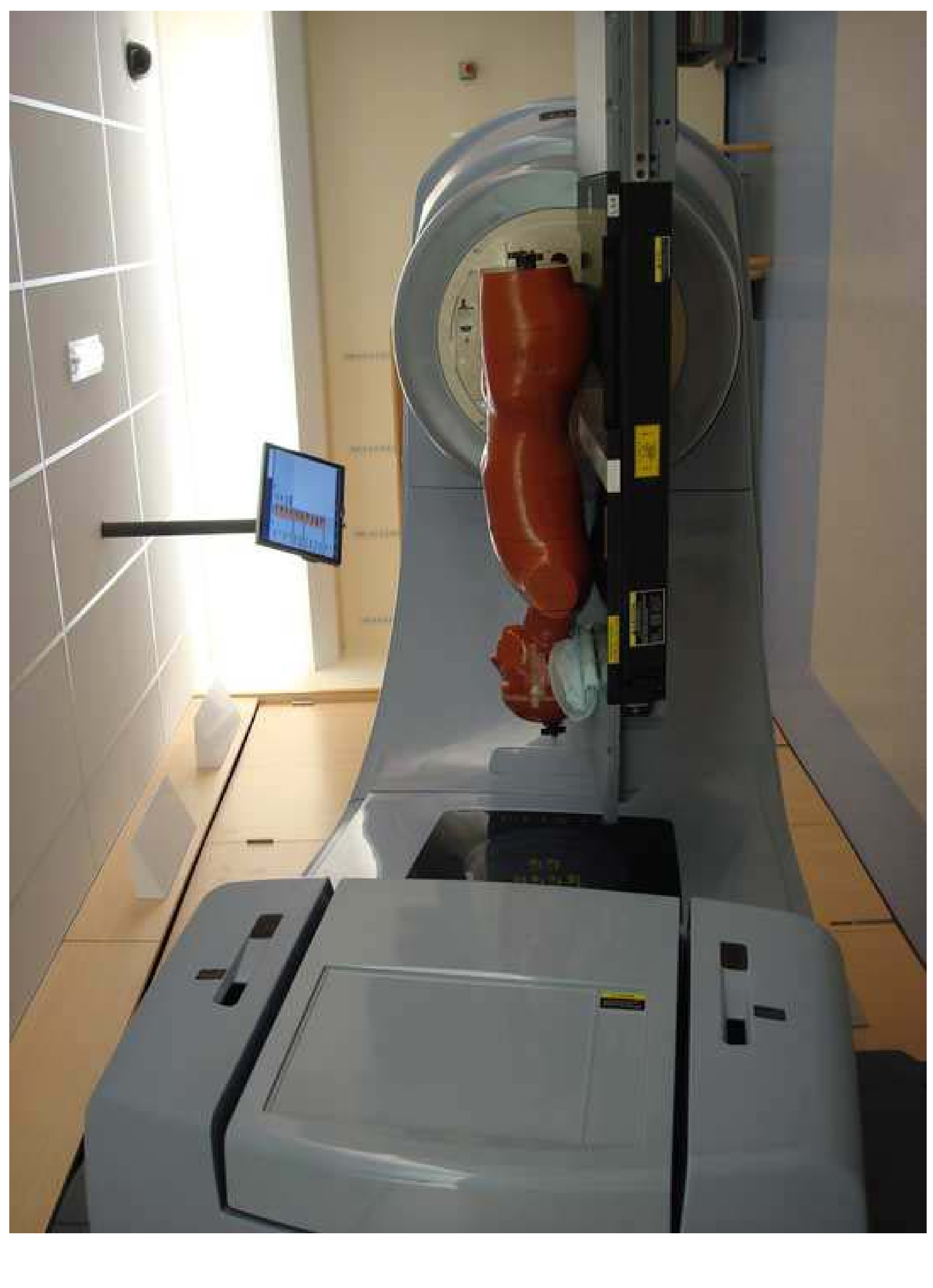




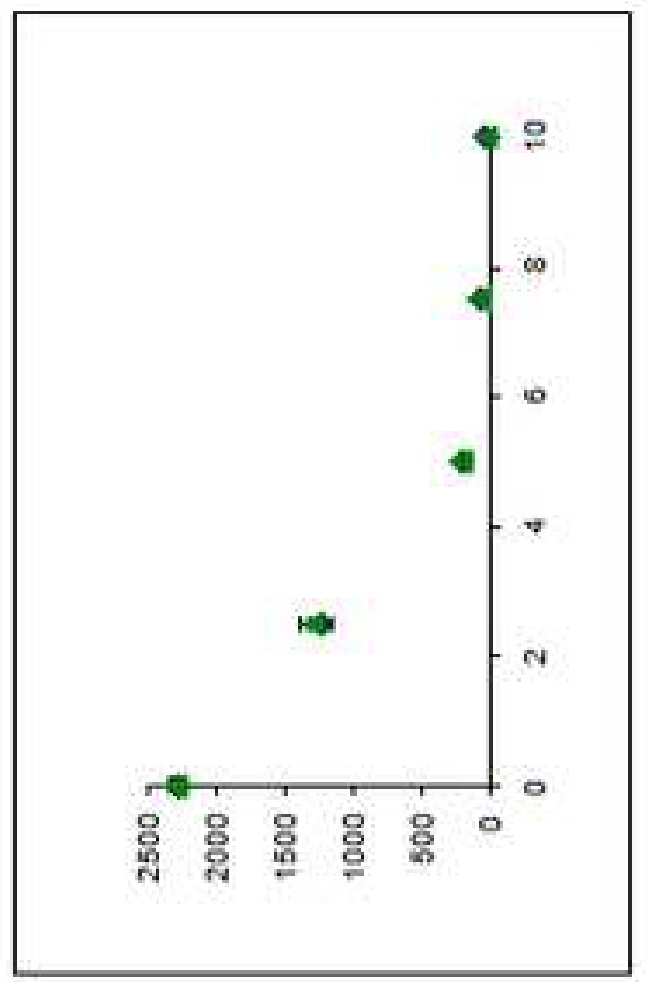

0
0
0
0

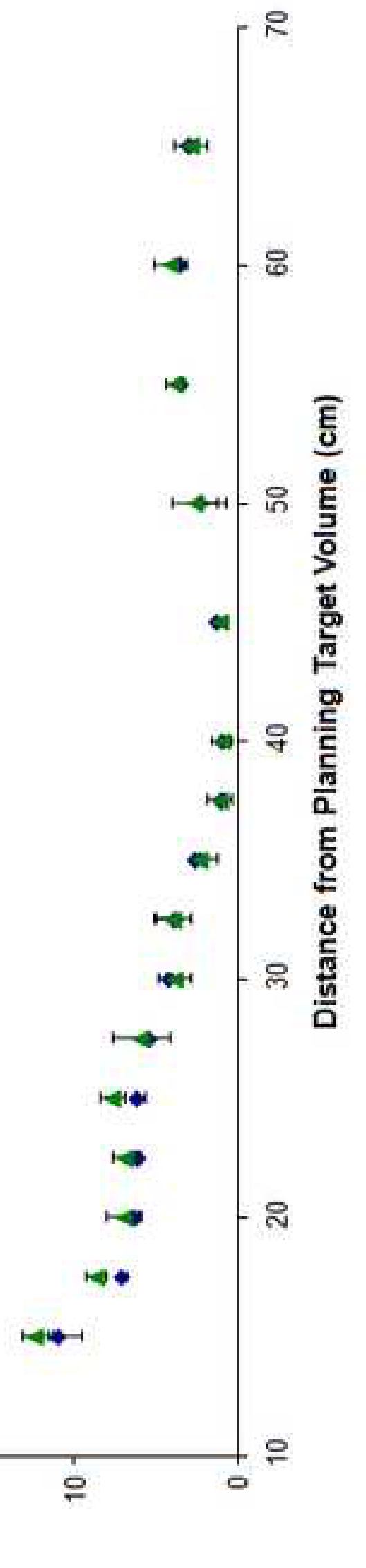



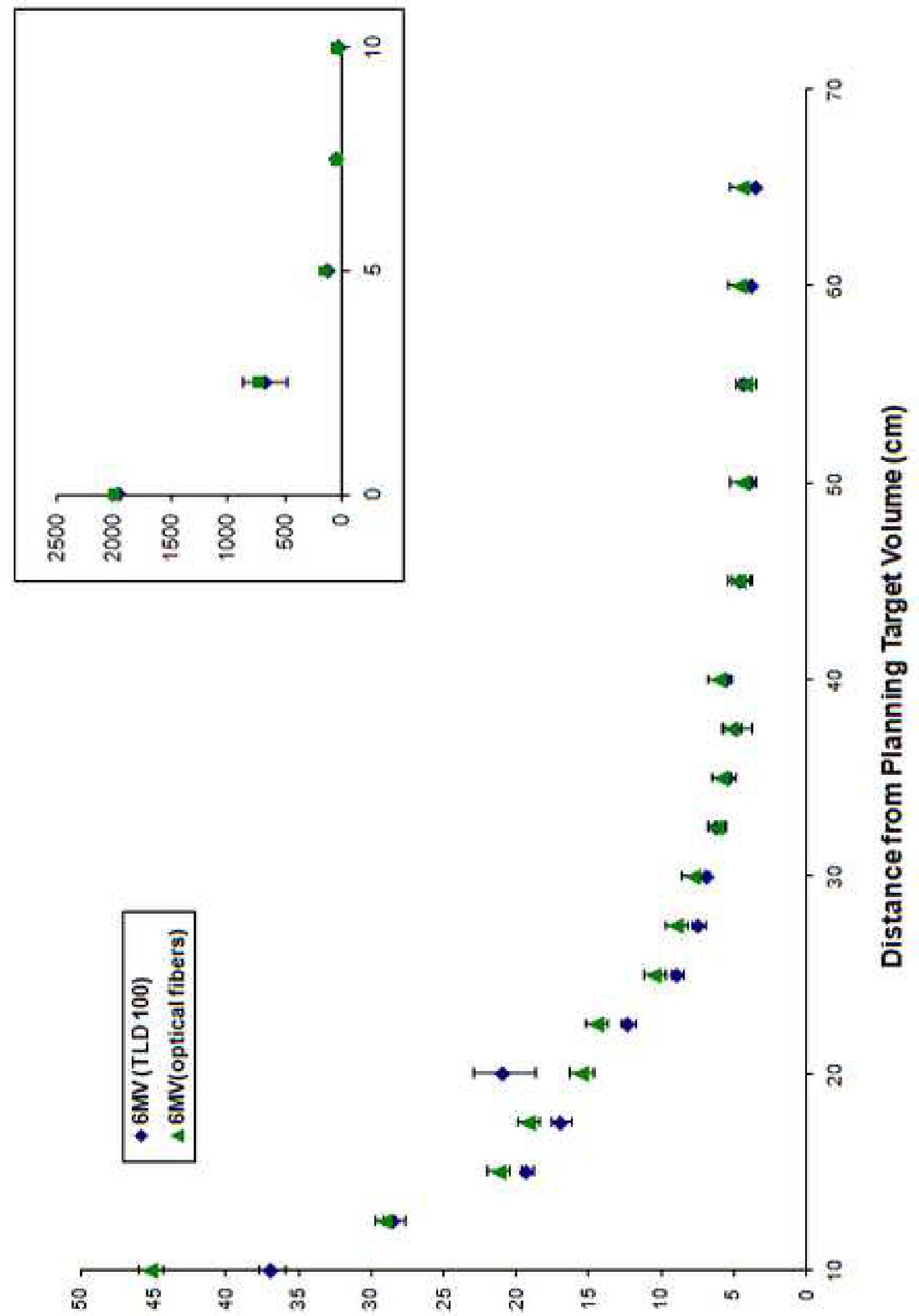

(Nou) อsog abesอn 


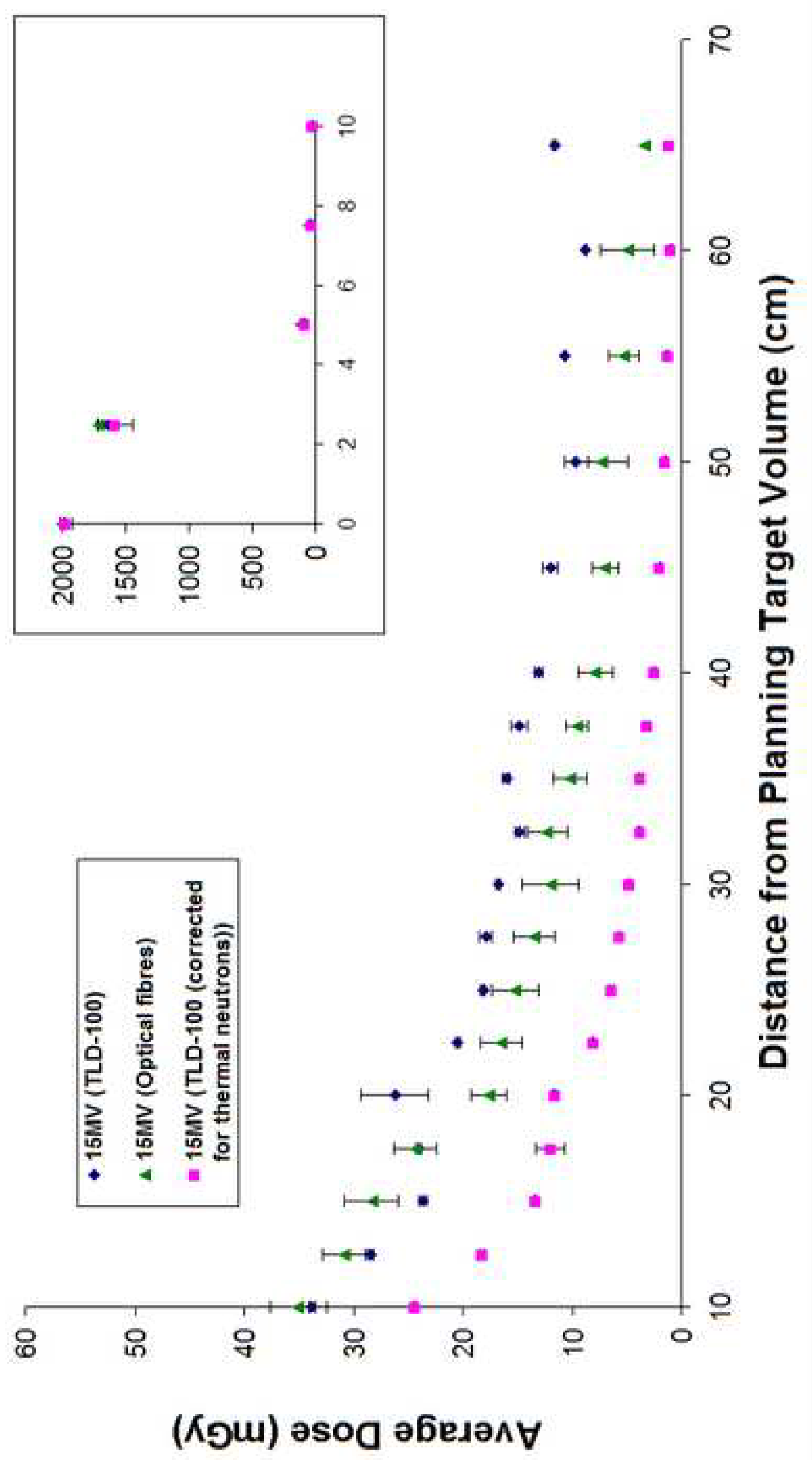




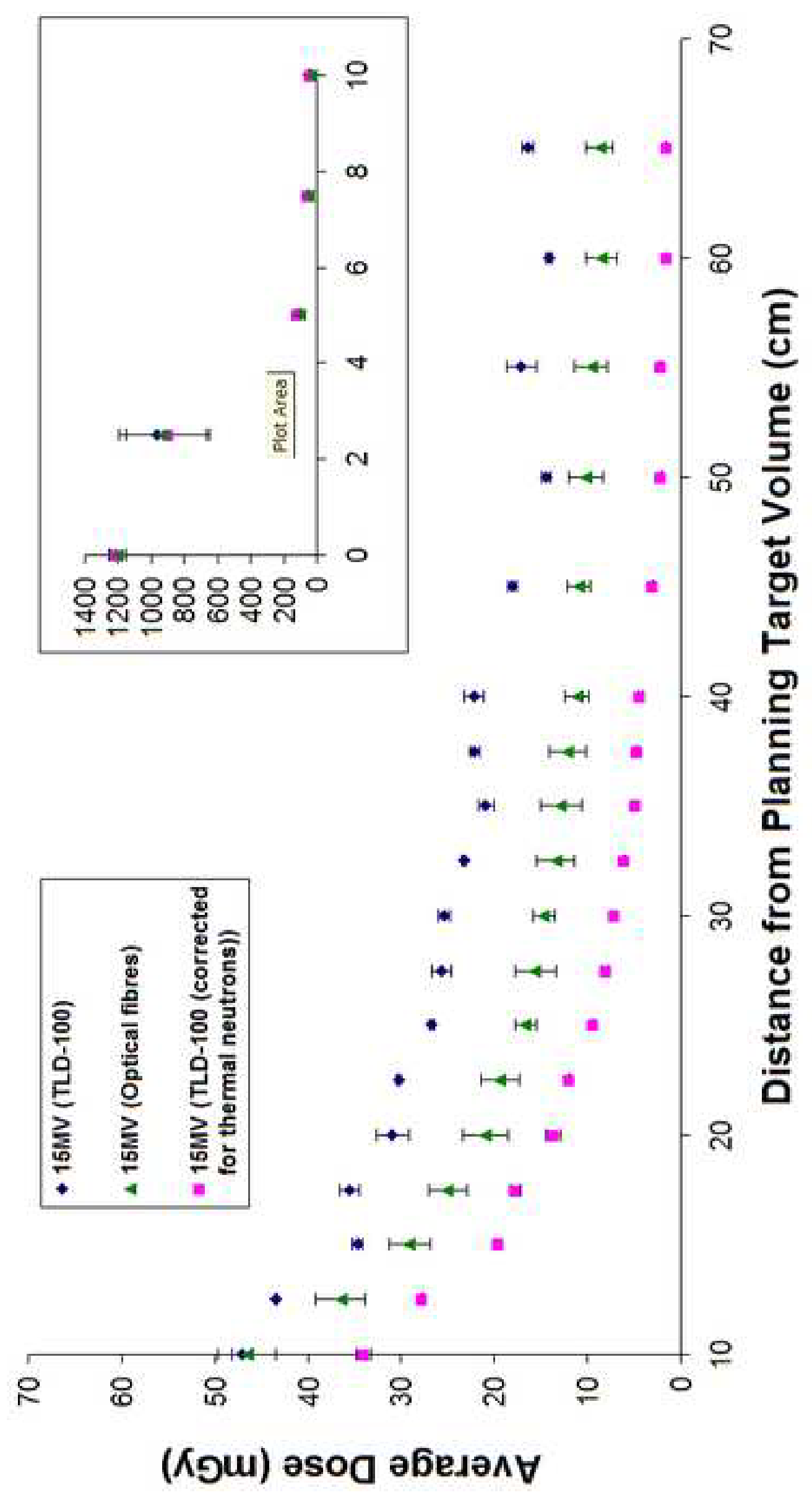

